\title{
In Situ Phase Transformation of Monodisperse Manganese Oxide Nanoparticles
}

\author{
Bahareh Deljoo ${ }^{1,2 *}$, Steven L. Suib ${ }^{1,2,3}$, Mark Aindow ${ }^{1,2}$ \\ 1. Dept. of Materials Science and Engineering, University of Connecticut, Storrs, CT 06269, USA \\ 2. Institute of Materials Science, University of Connecticut, Storrs, CT 06269, USA \\ 3. Dept. of Chemistry, University of Connecticut, Storrs, CT 06269, USA \\ *Corresponding author: Bahareh.deljoo@uconn.edu
}

The oxides of manganese are of special interest due to their multi-valent characteristics, low toxicity, and low cost [1]. These materials have been used in various areas including catalysts and batteries. In each of these cases, a particular phase of manganese oxide is most suitable; as such, understanding the phase transformations that occur in these oxides upon heating is important [2]. It is very challenging to develop an understanding of the phase transformations for manganese oxide nanoparticles, because of the length scale on which these processes occur. However, recent developments in micro-electro-mechanical system (MEMS) holders for in situ TEM experiments have enabled researchers to investigate the dynamic evolution of microstructures at high spatial resolution under a wide variety of different environmental conditions including heating.

In this work monodisperse amorphous manganese oxide nanoparticles were synthesized following the synthesis procedure reported by Soejima et al. [3]. The synthesis parameters were optimized for this study to obtain electron-transparent nanoparticles. The wide-angle XRD data from the as-synthesized sample confirmed that the powder was fully amorphous. The microstructure of this sample was studied using an FEI Teneo LoVac FEG-SEM and an FEI Talos F200X FEG-TEM. Examples of the secondary electron SEM and BF-TEM images obtained are shown in Figures 1(a) and (b). These images show that the sample is composed of monodisperse manganese oxide nanoparticles, with an average diameter of $250 \mathrm{~nm}$, which is suitable for direct in situ TEM investigations without requiring thinning of the samples. The corresponding selected area diffraction pattern (SADP) from the TEM specimen is shown in Figure 1(c), and this pattern exhibits only diffuse amorphous rings as expected.

The temperatures at which one would expect phase transformations to occur were identified by performing differential scanning calorimetry under argon from room temperature to $1200^{\circ} \mathrm{C}$, and two main processes were detected: one at around $500{ }^{\circ} \mathrm{C}$ and one at $900{ }^{\circ} \mathrm{C}$. In the in situ TEM experiments, the manganese oxide nanoparticles were transferred to an FEI Nano-Ex/iV heating stage and isothermal heat treatments were performed at temperatures below and above these transformation temperatures. The BF-TEM images and SADPs of the manganese oxide nano-particles show that they remain amorphous up to $400{ }^{\circ} \mathrm{C}$, whereupon the first sharp diffraction rings start to form. These rings become better defined with increasing temperature up to around $650^{\circ} \mathrm{C}$, corresponding to the completion of the first phase transformation. Indexing of the rings shows that this corresponds to crystallization of the amorphous manganese oxide in the form of nano-crystalline $\mathrm{MnO}$ (rock salt structure). Upon further heating to $900{ }^{\circ} \mathrm{C}$, additional diffraction spots appear in the SADPs, which suggest that part of the sample has transformed to coarser grains or particles of $\mathrm{Mn}_{2} \mathrm{O}_{3}$. This result was not anticipated, both because $\mathrm{Mn}_{2} \mathrm{O}_{3}$ corresponds to a higher oxidation state for $\mathrm{Mn}(3+$ vs. $2+$ for $\mathrm{MnO})$, and because of discrepancies with ex situ data. A possible explanation is suggested by changes that occur upon further heating whereby the manganese oxide reacts with the silicon nitrate substrate as shown in Figure 3. 


\section{References:}

[1] A Khan et al., J. Mater. Chem. A 6 (2018) p. 1590-1600.

[2] S Sultana et al., Catalysis Today 307 (2018) p. 20-28.

[3] T Soejima et al., Journal of Colloid and Interface Science 510 (2018) p. 272-279.

[4] This work was supported in part by the US DOE, under grant DE-FG02-86ER13622.A000, and by a research grant from Thermo Fisher Scientific. These studies were performed in the UConn/Thermo Fisher Center for Advanced Microscopy and Materials Analysis (CAMMA).
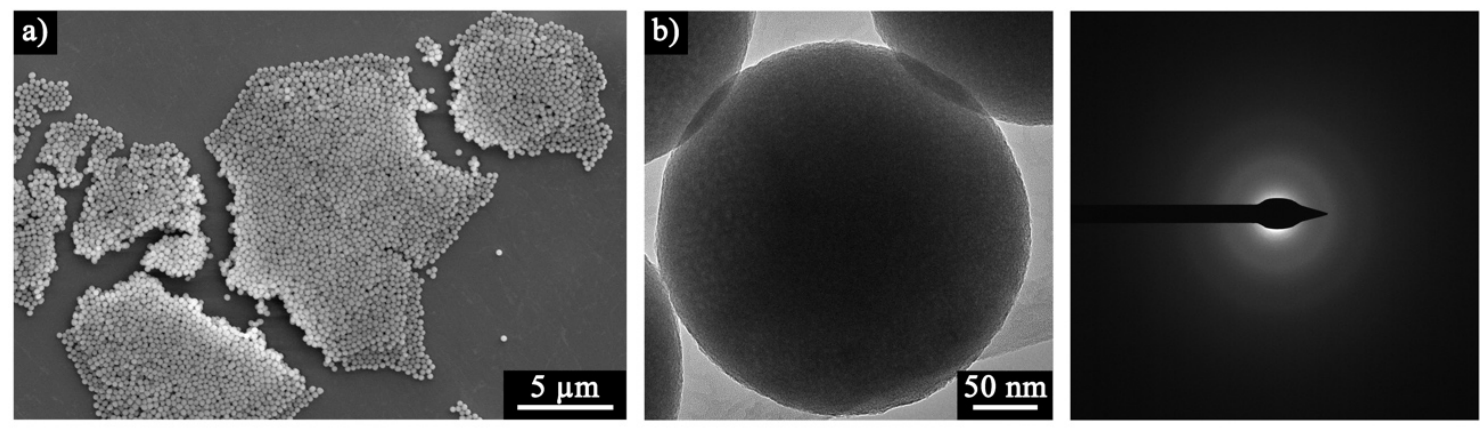

Figure 1. a) SEM, b) BF-TEM, and c) SADP of as-synthesized monodisperse manganese oxide.
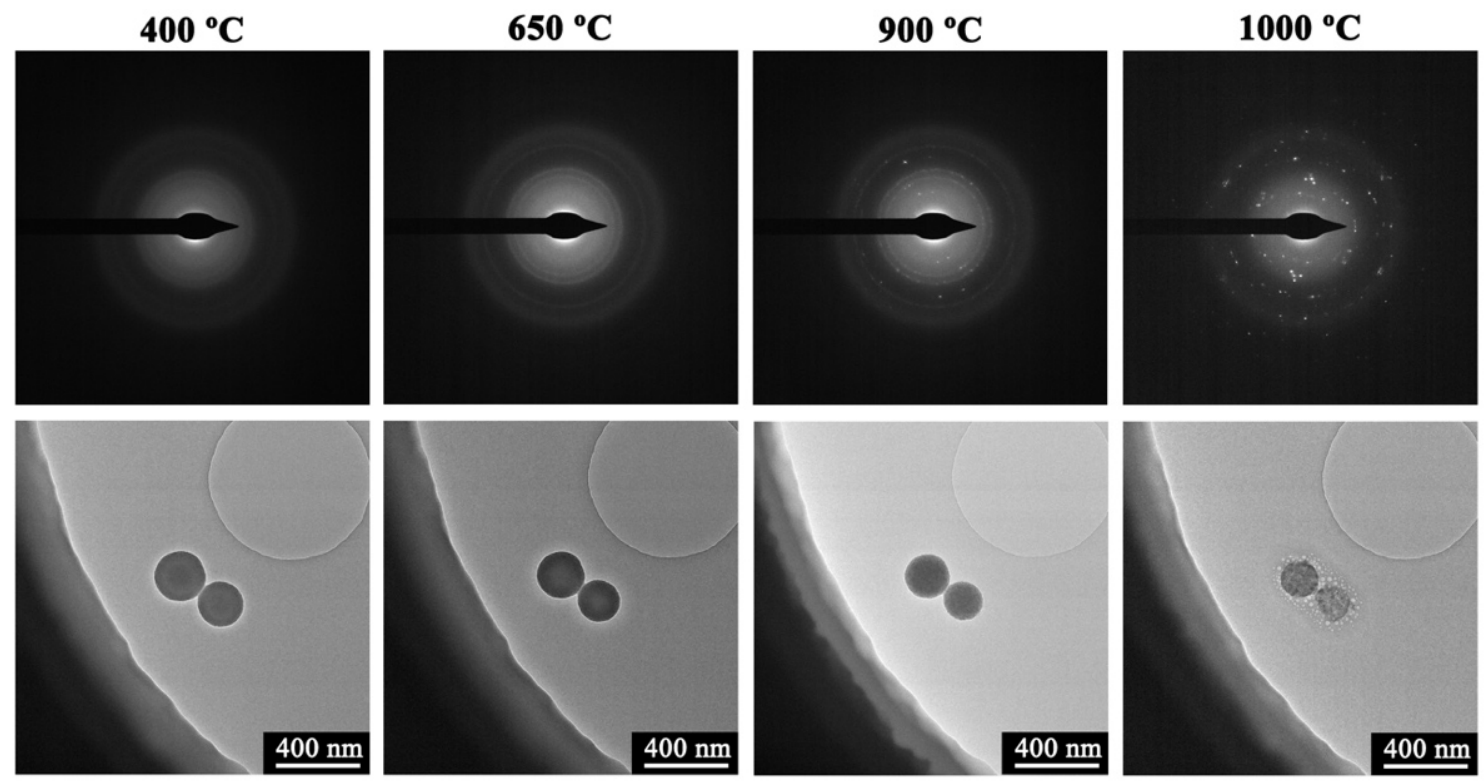

Figure 2. In situ TEM SADPs and BF images of manganese oxide nanoparticles.
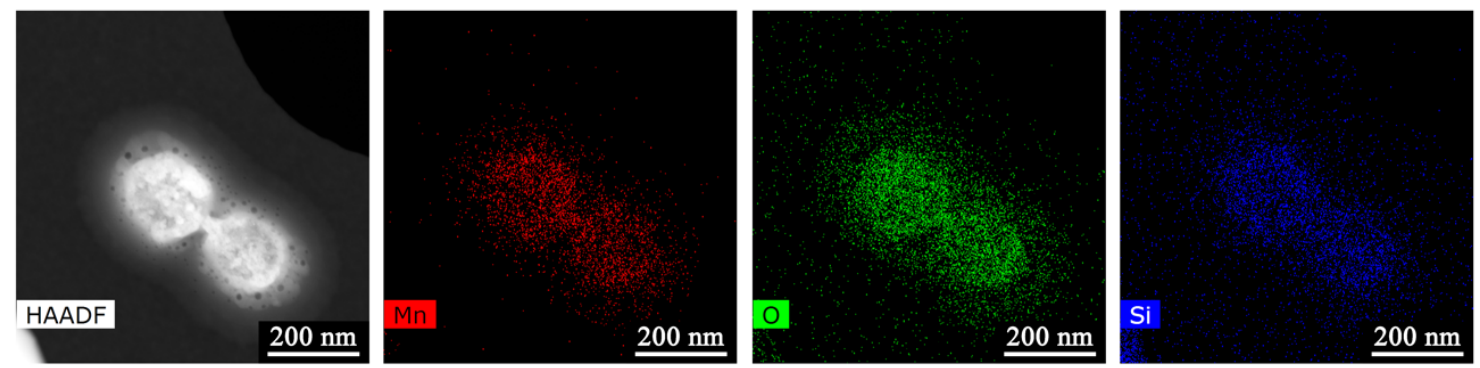

Figure 3. STEM data obtained from the sample heated to $1000{ }^{\circ} \mathrm{C}$ as shown in Figure 2 above. The Xray maps show the incorporation of $\mathrm{Si}$ into the manganese oxide nanoparticles. 